\title{
Astronomical Photographs.
}

$W^{E}$ directed attention some time ago to the excellent collection of astronomical photographs issued in various forms by the University of

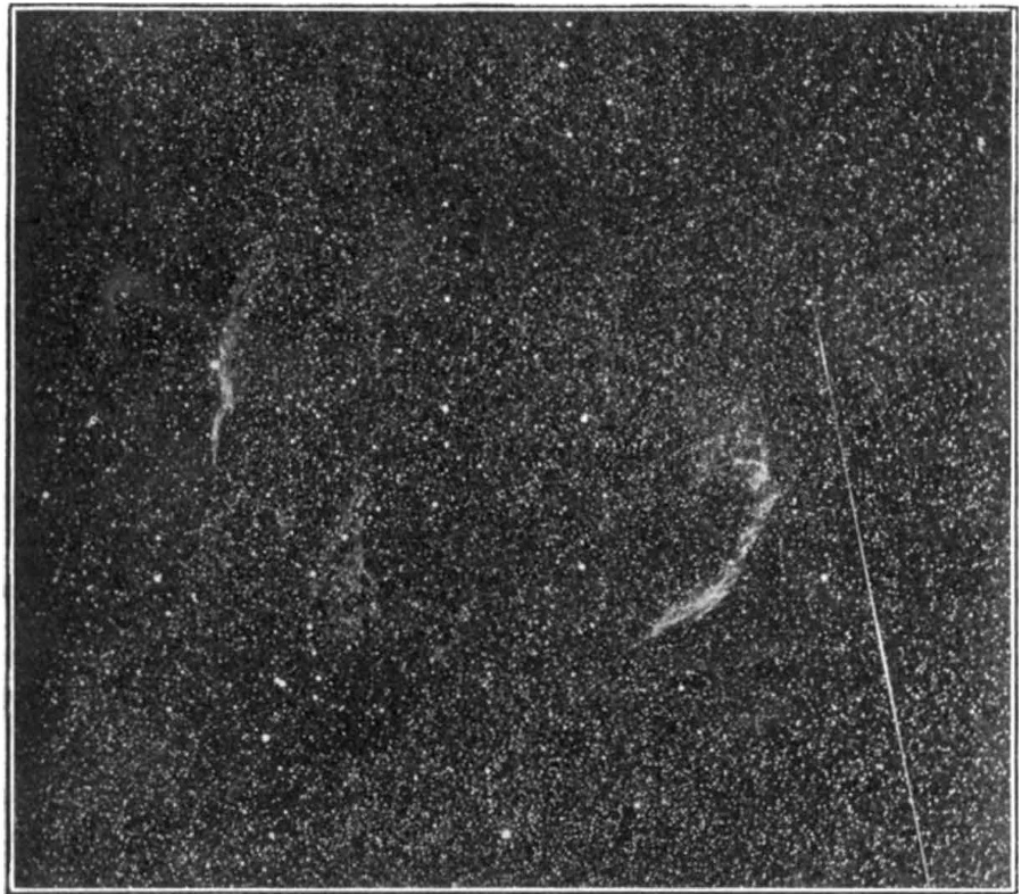

Fig. 1,-Extended gaseous nebula in Cygnus. Photographed at the Yerkes Observatory.
2 dollars for size 8 in. $\times$ ro in. to ro dollars for size 18 in. $\times 22$ in. Card descriptions of the hundred selected photographs are 5 cents each, or 2.50 dollars for the set.

There is no need to emphasise the excellence of the illustrations themselves-the work of the Yerkes Observatory is too well known for that-and we can perhaps give the reproductions no higher praise than by saying that, so far as we can judge from the specimens we have seen, they are in every respect worthy of the originals. They cover the whole range of descriptive astronomy, and have evidently been chosen with the greatest care. The selected list of roo, for example, comprises various types of solar phenomena; the moon at various ages (including earth-shine on the moon, a total eclipse, and an occultation of Aldebaran); the whole of the planets, including trails of asteroids; a good selection of comets and a meteor-trail; star fields, showing conspicuous constellations, circumpolar and equatorial star-trails, variable, double and temporary stars ; open and globular star clusters ; nebulæ of all typesdiffuse (bright and dark), planetary,

Chicago Press for the benefit particularly of lecturers annular and spiral; star clouds of the Milky and teachers. We have now received a complete set 1 Way; solar, stellar, nebular, and cometary spectra, of card descriptions of one hundred of the photographs, specially selected "for the benefit of institutions or individuals desiring a small collection thoroughly representative of present astronomical science," and we take this opportunity of making known as widely as possible the admirable work which is in this way being done to popularise interest in astronomy.

The photographs, nearly all of which were taken at the Yerkes Observatory, are issued in the form of lantern slides, transparencies, and prints. The lantern slides, which are $4 \times 3 \frac{1}{4}$ in. in size and are therefore unfortunately unsuitable for the ordinary carriers of British lanterns, are supplied at 75 cents each, but the total cost of the selected set of roo slides is 62.50 dollars, involving a saving of approximately $\mathbf{2}$ dollars. Unmounted prints,

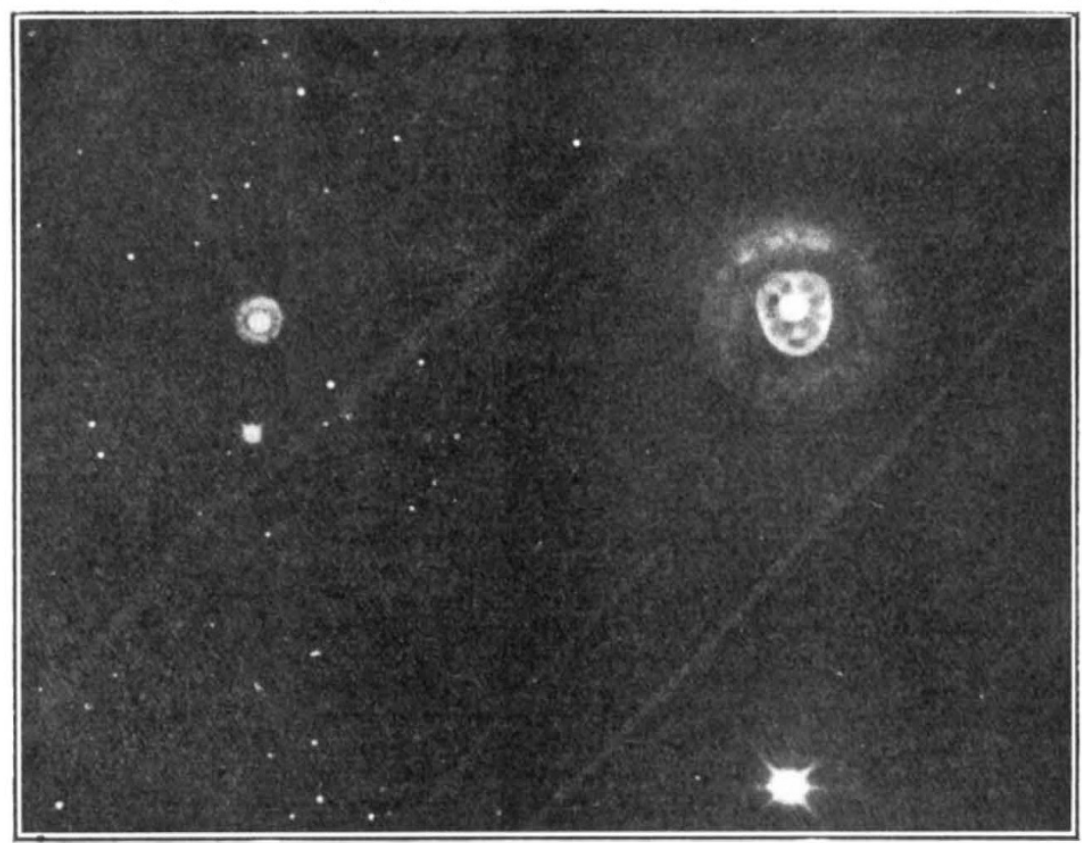

(I)

FIG. 2.-Planetary nebula in Gemini. Photographed at the Mount Wilson Observatory (x) Showirg structure of outer ring; (2) showing inner structure.

matt or glossy, are 50 cents each; transparencies, which | showing the chief stellar types and the spectra of are made to order in five sizes, vary in price from spectroscopic binaries and of a nova; and finally,

NO. 2840 , VOL. I I 3$]$ 
views of the Yerkes Observatory and the chief instruments in use there. Portraits of about fifty astronomers are also included.

It will be realised from this summary that a very

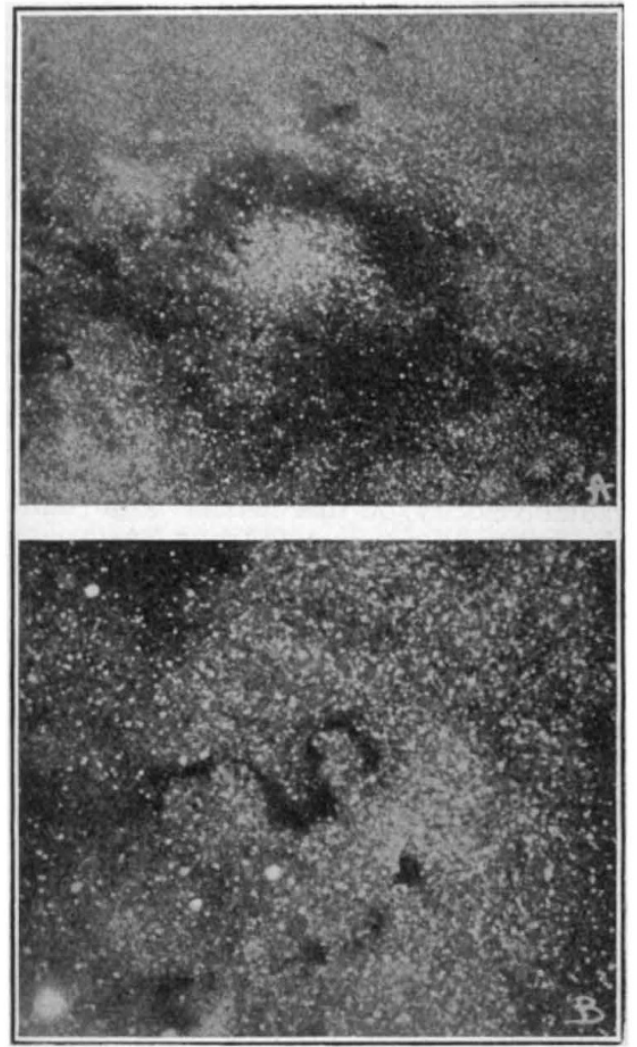

FIG. 3.-Dark nebulæ in the Milky Way. Photographed at the Yerkes Observatory.

The upper picture shows very large curved marking with definite boundaries, and the lower shows an S-shaped marking, as well as several small dark spots south of it. Although formerly believed to be vacancies, these objects are now accepted by many astronomers as representing actually very faintly luminous or non-luminous masses.

wide field has been covered, and that the collection is truly representative. It becomes clear, however, on a careful examination of the catalogues, that the selectors have done their work more efficiently than a mere summary of the slides would indicate. The total number of illustrations issued now amounts to more than 800 , and in making a selection of only roo, many extremely useful slides have had to be omitted. This disadvantage has been to a considerable extent overcome by selecting slides suitable for more than one purpose. For example, no slide intended to illustrate the determination of radial velocities is included, but there are two spectra of $\mu$ Orionis-a spectroscopic binary of which only one component yields a spectrumwhich are perfectly suitable for the purpose. (These spectra, we may remark, will be useful also to advanced students, as providing an exercise in measurement.) Again, there is only one picture described as a " meteortrail," but among the illustrations of nebulæ there is one which includes a meteor-trail also. We have no hesitation in saying that the lecturer or teacher who carefully studies these selected illustrations will find them to cover a much wider range than their number and titles would indicate.
The descriptions of the slides are clearly printed on cards 6 in. $\times 4$ in. in size, and are each about 250 words in length. They are clear and simple, and not only point out the chief features of the slides they are intended to describe, but include also a considerable amount of interesting matter relating to the general types of the bodies to which they refer. More than half of the descriptive cards were written by Prof. Frost, about a quarter of them by Prof. Barrett, and a dozen or so by Prof. Barnard. It is inevitable, in view of the very rapid rate at which astronomy is now advancing, that some of the descriptions should be slightly out-of-date. Thus, for example, we are told that "the best estimates of the extent of the Milky Way place it at about 30,000 light-years." In view of Shapley's work, this is now at least doubtful. On the whole, matters of controversy have been avoided, though we note that the existence of "nebulium" is assumed. As the publishers point out, the descriptions, consisting of nearly 24,000 words, are equivalent to an octavo volume of about 70 pages. While they are not

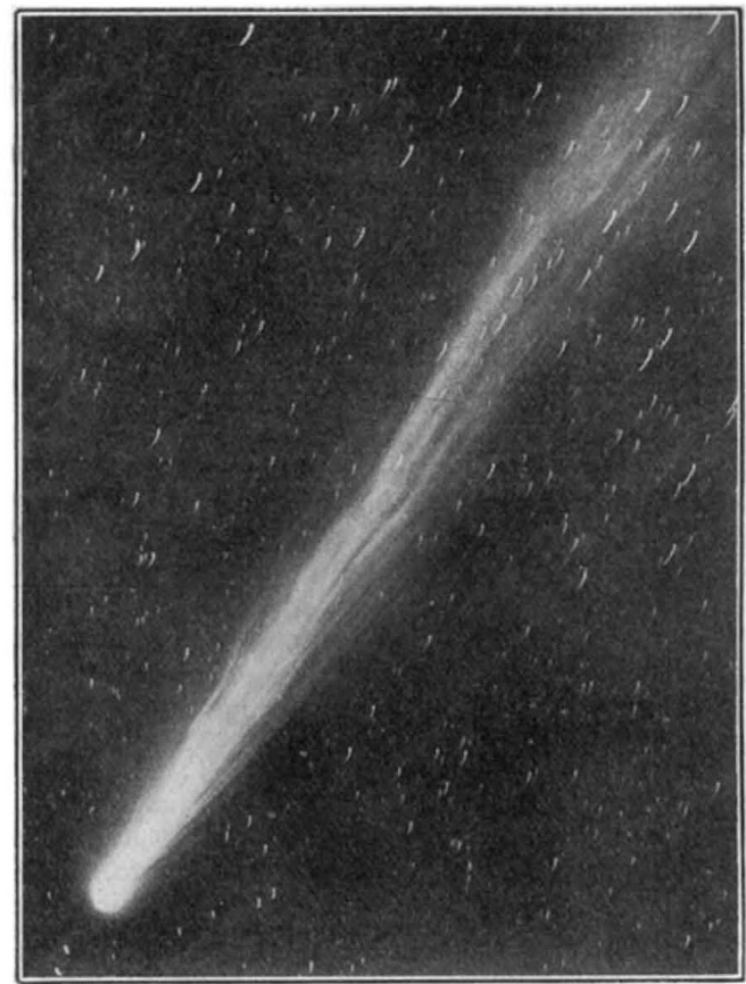

FiG. 4.-Comet Brooks, October 23, 19xx. Photographed at the Yerkes Observatory.

Although this comet was visible to the naked eye and had a tail $26^{\circ}$ long, none of the details shown in the illustration could be seen either with the naked eye or with a telescope. The head of the comet was about 300,000 miles in diameter at the time when the photograph was taken.

recommended as a textbook, they would be in many respects the equivalent of such a book if they were accompanied by prints from the negatives which the slides represent. We give the following descriptions of two of the four illustrations which are here reproduced, in order to show their general character. These descriptions are quite typical, and give a true idea of the quality of the whole. 
N r6. Extended Gaseous Nebul In Cygnus. Photographed by Prof. E. E. Barnard with the Bruce Telescope.

These two wonderful filamentary nebulæ are parts of a still larger ring, the fainter portions of which can be photographed, but probably have never been seen with the eye. They are sometimes called the " cirrus nebula " because of their resemblance to the cirrus clouds frequently seen in the sky. They are gaseous, composed of nebulium, hydrogen, and helium. They are of enormous size, each of the two brighter nebulæ being over a degree in length, and even the slenderest filaments must be hundreds of millions of miles in width.

It will be noticed that the nebula at the left of the slide lies along the line of separation of a region thick with stars from a region noticeably less dense in stars. It has not yet been possible to detect any motion of these filaments with respect to the stars, but probably it will ultimately be measured when photographs are available separated by a long period of years.

During this long exposure of nearly six hours, a meteor flashed across this part of the sky, and its impression was caught on the plate. It is brighter than some of the filaments of the nebulæ, but there could be no greater contrast in actual size or brightness, for the meteor was only a small pebble, or perhaps a grain of sand, burning itself out by friction in our atmosphere, and probably less than roo miles away, while the nebula is several hundred light-years distant from us.

N 5 I. Planetary Nebula in Gemini, N.G.C. 2392. Photographed by Prof. E. E. Barnard.

Planetary nebulæ, of which there are only about I50 catalogued, are all apparently small in size, are rather dense, and in general have well-defined outlines resembling ellipsoidal shells, with a Wolf-Rayet star at the centre. They are uniformly gaseous, their inner parts containing helium, nebulium, and hydrogen; their outer parts, hydrogen only.
Since they have a tendency to lie in the plane of the Milky Way, they probably belong to our own system. One planetary nebula has been found to be distant I 40 light-years, but the average distance is probably about Iooo light-years. From rather uncertain data some are found to be rotating about an axis with periods of from 600 to 14,000 years, their masses being three to one hundred times that of the sun, but with densities of about one-millionth that of our atmosphere.

The two photographs are of the same nebula, but taken with the 25-foot and the Ioo-foot focus, respectively, of the 6o-inch reflector at Mount Wilson Observatory. The first is over-exposed in the centre in order to bring out the details of the faint outer ring, while the one of large scale shows the inner structure. The appearance of this nebula is unique, and bears little resemblance to that of other nebulæ.

The fact that planetary nebulæ have an average radial velocity of 24 miles per second discountenances the theory that they condense into the early types of stars, $\mathrm{O}$ and $\mathrm{B}$, whose velocities are markedly less. The radial velocity of this nebula is 50 miles per second. It has been suggested that planetary nebulæ are the wrecks of ancient collisions among stars.

We hope that the effort which the publishers of these illustrations and descriptions are making to spread the knowledge of astronomy in so interesting a manner will meet with the success which it deserves. It will be understood that the work of distributing lantern slides and photographs, or descriptions of them, is not a matter of business with the Yerkes Observatory, as the proceeds from sales cannot do more than cover the cost of manufacture, quite apart from the time spent by scientific members of the staff of the Observatory in selecting the subjects, supervising the photographic work, and writing the descriptions.

Copies of an illustrated catalogue of the photographs made at the Yerkes Observatory, or of a selected list of one hundred slides, may be obtained from the University of Chicago Press, Chicago, Illinois, U.S.A.

\section{Brain and Speech.}

\section{By Tudor Jones, M.B.}

$A \mathrm{~S}$ the conclusions which Dr. Henry Head bases upon his extensive researches into the phenomena of aphasia are now approaching a comprehensive form, ${ }^{1}$ the present is a convenient time to attempt a summary of his views. Fourteen years ago, in the light of his work on the part played by the brain cortex in sensation, it became clear that new tests must be devised before the clinical investigation of aphasia could produce any satisfactory result.

It is less necessary to describe here the elaborate series of tests which Dr. Head invented, every one of them new or used in a new way, than to direct attention to the principles which guided him in this important part of his work. ${ }^{2}$ Foremost among these was the conception, which a study of his own work and that of Sherrington and his collaborators led Head to formulate, that cortical injury, so far from removing a strictly definable anatomical "centre," disturbs a highly organised act, the very variable end-point of a whole march of events occurring in the central nervous

' "Speech and Cerebral Localisation," by Henry Head, Brain, vol. xivi. Part iv., r923 (London: Macmillan and Co., Ltd.) 6s. net.

${ }^{2} \mathrm{~A}$ description of the tests appeared in Brain, vol. xliii., 1920. system. The effects of a destructive lesion of the brain cortex are all negative. They do not reveal the primitive elements out of which speech issues, nor do they break it up in accordance with our conceptual terms in describing it. What they do is to prevent, in part, the activity of a highly organised group of functions all at the cortical level. Head insists very strongly upon the negative characters of this interruption, distinguishing them from such positive effects as the spasticity of hemiplegia, which reveals the uncontrolled activity of neurons at a different level. Thus his first aim was to determine with the greatest possible accuracy what it was that the patient could not do, or could do only sometimes or with difficulty. The tests at length devised are not considered to be complete or incapable of improvement; but that they constitute in themselves a great advance in clinical method has been admitted even by those who, for the present, are unable to adopt Head's more theoretical conclusions.

This great gain is, however, less than that which must proceed from due observance of the principle itself: that what we must strive to ascertain is not the defect 